\title{
The natural operators of general affine connections into general affine connections
}

\begin{abstract}
We reduce the problem of describing all $\mathcal{M} f_{m}$-natural operators transforming general affine connections on $m$-manifolds into general affine ones to the known description of all $G L\left(\mathbf{R}^{m}\right)$-invariant maps $\mathbf{R}^{m *} \otimes \mathbf{R}^{m} \rightarrow$ $\otimes{ }^{k} \mathbf{R}^{m *} \otimes \otimes{ }^{k} \mathbf{R}^{m}$ for $k=1,3$.
\end{abstract}

Introduction. All manifolds considered in this paper are assumed to be finite dimensional, without boundaries, second countable, Hausdorff and smooth (of class $C^{\infty}$ ). Maps between manifolds are assumed to be smooth (of class $C^{\infty}$ ). The category of $m$-dimensional manifolds and their embeddings is denoted by $\mathcal{M} f_{m}$.

A classical linear connection on a manifold $M$ is a right invariant connection $\Gamma$ on the principal fiber bundle $L M$ of linear frames of $M$. It can be considered equivalently as the corresponding R-bilinear map $\nabla: \mathcal{X}(M) \times$ $\mathcal{X}(M) \rightarrow \mathcal{X}(M)$ such that $\nabla_{f X} Y=f \nabla_{X} Y$ and $\nabla_{X} f Y=X(f) Y+f \nabla_{X} Y$ for any map $f: M \rightarrow \mathbf{R}$ and any vector fields $X, Y \in \mathcal{X}(M)$ on $M$, see [2].

A general affine connection on $M$ is a right invariant connection $\Gamma$ on the principal fiber bundle $A M$ of affine frames of $M$. It can be equivalently considered as the corresponding pair $(\nabla, K)$ consisting of a classical linear connection $\nabla$ on $M$ and a tensor field $K$ of type $(1,1)$ on $M$, see [2].

The general concept of natural operators can be found in [3].

2010 Mathematics Subject Classification. 58A32.

Key words and phrases. General affine connection, natural operator. 
In the present note, we study the problem of finding all $\mathcal{M} f_{m}$-natural operators $B: Q_{\text {gen-af }} \rightsquigarrow Q_{\text {gen-af }}$ transforming general affine connections $(\nabla, K)$ on $m$-manifolds $M$ into general affine connections $B(\nabla, K)$ on $M$.

Given an $\mathcal{M} f_{m}$-natural operator $B: Q_{\text {gen-af }} \rightsquigarrow Q_{\text {gen-af }}$, we define an $\mathcal{M} f_{m}$-natural operator $\Delta: Q_{\text {gen-af }} \rightsquigarrow\left(\otimes^{2} T^{*} \otimes T\right) \oplus\left(T^{*} \otimes T\right)$ by

$$
B(\nabla, K)=(\nabla, K)+\Delta(\nabla, K)
$$

for all general affine connections $(\nabla, K)$ on $m$-manifolds $M$, and vice versa. So, to find all $\mathcal{M} f_{m}$-natural operators $B: Q_{\text {gen-af }} \rightsquigarrow Q_{\text {gen-af }}$ it is sufficient to find all $\mathcal{M} f_{m}$-natural operators $\Delta: Q_{\text {gen-af }} \rightsquigarrow\left(\otimes^{2} T^{*} \otimes T\right) \oplus\left(T^{*} \otimes T\right)$ transforming general affine connections $(\nabla, K)$ on $m$-manifolds $M$ into pairs $\Delta(\nabla, K)=\left(\Delta^{1}(\nabla, K), \Delta^{2}(\nabla, K)\right)$ of tensor fields $\Delta^{1}(\nabla, K)$ of type $(1,2)$ and $\Delta^{2}(\nabla, K)$ of type $(1,1)$ on $M$.

In the present note, we prove that the above problem of finding all $\mathcal{M} f_{m^{-}}$ natural operators $B: Q_{\text {gen-af }} \rightsquigarrow Q_{\text {gen-af }}$ (or $\Delta: Q_{\text {gen-af }} \rightsquigarrow\left(\otimes^{2} T^{*} \otimes T\right) \oplus$ $\left.\left(T^{*} \otimes T\right)\right)$ can be reduced to the one of describing all $G L\left(\mathbf{R}^{m}\right)$-invariant maps $\mathbf{R}^{m *} \otimes \mathbf{R}^{m} \rightarrow \otimes^{k} \mathbf{R}^{m *} \otimes \otimes{ }^{k} \mathbf{R}^{m}$ for $k=1,3$.

This "reduction" is satisfactory, because the $G L\left(\mathbf{R}^{m}\right)$-invariant maps $\mathbf{R}^{m *} \otimes \mathbf{R}^{m} \rightarrow \otimes{ }^{k} \mathbf{R}^{m *} \otimes \otimes{ }^{k} \mathbf{R}^{m}$ for $k=1,2,3$ are described in [1].

1. The crucial lemma. We prove the following lemma.

Lemma 1. There is the bijection between the set $C$ of all $\mathcal{M} f_{m}$-natural operators $\Delta: Q_{\text {gen-af }} \rightsquigarrow\left(\otimes^{2} T^{*} \otimes T\right) \oplus\left(T^{*} \otimes T\right)$ and the set $D$ of all $G L\left(\mathbf{R}^{m}\right)$ invariant maps $\left(\bigwedge^{2} \mathbf{R}^{m *} \otimes \mathbf{R}^{m}\right) \oplus\left(\mathbf{R}^{m *} \otimes \mathbf{R}^{m}\right) \oplus\left(\otimes^{2} \mathbf{R}^{m *} \otimes \mathbf{R}^{m}\right) \rightarrow\left(\otimes \mathbf{R}^{m *} \otimes\right.$ $\left.\mathbf{R}^{m}\right) \oplus\left(\mathbf{R}^{m *} \otimes \mathbf{R}^{m}\right)$.

Proof. We define a map $\Phi: C \rightarrow D$ as follows.

Any $\Delta \in C$ is determined by the values

$$
\begin{aligned}
\Delta(\nabla, K)(x) & =\left(\Delta^{1}(\nabla, K)(x), \Delta^{2}(\nabla, K)(x)\right) \\
& \in\left(\otimes^{2} T_{x}^{*} M \otimes T_{x} M\right) \oplus\left(T_{x}^{*} M \otimes T_{x} M\right)
\end{aligned}
$$

for all $m$-manifolds $M$, all linear connections $\nabla$ on $M$, all tensor fields $K$ of type $(1,1)$ on $M$ and all $x \in M$. Because of the $\mathcal{M} f_{m}$-invariance of $\Delta$, we may assume that $M=\mathbf{R}^{m}, x=0$. We can even assume that $i d_{\mathbf{R}^{m}}$ is $\nabla$-normal with center 0 (then $\nabla(0) \in \bigwedge^{2} \mathbf{R}^{m *} \otimes \mathbf{R}^{m}$ because the Christoffel symbols $\nabla_{j k}^{i}$ of $\nabla$ satisfy $\left.\nabla_{j k}^{i}(0)+\nabla_{k j}^{i}(0)=0\right)$. Then using the invariance of $\Delta$ with respect to the homotheties $a_{t}=t i d_{\mathbf{R}^{m}}$ for $t>0$, we obtain the homogeneity condition

$$
\Delta\left(\left(a_{t}\right)_{*} \nabla,\left(a_{t}\right)_{*} K\right)(0)=\left(t \Delta^{1}(\nabla, K)(0), \Delta^{2}(\nabla, K)(0)\right) .
$$

Because of the homogeneous function theorem [3], this type of the homogeneity implies that $\Delta(\nabla, K)(0)$ depends on $\nabla(0)$ and $j_{0}^{1} K$ (only). Let $\left(\Lambda, \tau_{0}, \tau_{1}\right) \in\left(\bigwedge^{2} \mathbf{R}^{m *} \otimes \mathbf{R}^{m}\right) \oplus\left(\mathbf{R}^{m *} \otimes \mathbf{R}^{m}\right) \oplus\left(\otimes^{2} \mathbf{R}^{m *} \otimes \mathbf{R}^{m}\right) \tilde{=}\left(\bigwedge^{2} T_{0}^{*} \mathbf{R}^{m} \otimes\right.$ 
$\left.T_{0} \mathbf{R}^{m}\right) \oplus J_{0}^{1}\left(T^{*} \mathbf{R}^{m} \otimes T \mathbf{R}^{m}\right)$, where $\cong$ is the usual $G L\left(\mathbf{R}^{m}\right)$-invariant identification. We put

$$
\Phi(\Delta)\left(\Lambda, \tau_{0}, \tau_{1}\right):=\Delta(\nabla, K)(0) \in\left(\otimes^{2} \mathbf{R}^{m *} \otimes \mathbf{R}^{m}\right) \oplus\left(\mathbf{R}^{m *} \otimes \mathbf{R}^{m}\right)
$$

(modulo the usual $G L\left(\mathbf{R}^{m}\right)$-invariant identification), where $\nabla$ is the linear connection on $\mathbf{R}^{m}$ such that the Christoffel symbols of $\nabla$ with respect to the chart $i d_{\mathbf{R}^{m}}$ are constant maps and $\nabla(0)=\nabla^{o}(0)+\Lambda$ and $\nabla^{o}$ is the usual flat torsion free connection on $\mathbf{R}^{m}$ and $K$ is the tensor field of type $(1,1)$ on $\mathbf{R}^{m}$ such that the coefficients of $K$ in the chart $i d_{\mathbf{R}^{m}}$ are polynomials of degree not more than 1 and $j_{0}^{1} K=\left(\tau_{0}, \tau_{1}\right)$.

Since $\Delta$ is determined by $\Phi(\Delta), \Phi$ is injective.

It remains to show that $\Phi$ is surjective. Let $c:\left(\bigwedge^{2} \mathbf{R}^{m *} \otimes \mathbf{R}^{m}\right) \oplus\left(\mathbf{R}^{m *} \otimes\right.$ $\left.\mathbf{R}^{m}\right) \oplus\left(\otimes^{2} \mathbf{R}^{m *} \otimes \mathbf{R}^{m}\right) \rightarrow\left(\otimes{ }^{2} \mathbf{R}^{m *} \otimes \mathbf{R}^{m}\right) \oplus\left(\mathbf{R}^{m *} \otimes \mathbf{R}^{m}\right)$ be a $G L\left(\mathbf{R}^{m}\right)$ invariant map (an element from $D$ ). Using the usual $G L\left(\mathbf{R}^{m}\right)$-invariant identification $\mathbf{R}^{m}=T_{0} \mathbf{R}^{m}$, we have the $G L\left(\mathbf{R}^{m}\right)$-invariant map

$$
\begin{aligned}
c:\left(\bigwedge^{2} T_{0}^{*} \mathbf{R}^{m} \otimes T_{0} \mathbf{R}^{m}\right) \oplus & \left(J_{0}^{1}\left(T^{*} \mathbf{R}^{m} \otimes T \mathbf{R}^{m}\right)\right) \rightarrow \\
& \rightarrow\left(\otimes^{2} T_{0}^{*} \mathbf{R}^{m} \otimes T_{0} \mathbf{R}^{m}\right) \oplus\left(T_{0}^{*} \mathbf{R}^{m} \otimes T_{0} \mathbf{R}^{m}\right) .
\end{aligned}
$$

Let $(\nabla, K)$ be a general connection on an $m$-manifold $M$. Using $c$, we define a pair $\Delta_{c}(\nabla, K)$ consisting of tensor fields $\Delta_{c}^{1}(\nabla, K)$ of type $(1,2)$ and $\Delta_{c}^{2}(\nabla, K)$ of type $(1,1)$ on $M$ as follows. Let $x \in M$. Consider a normal coordinate system $\varphi$ of $\nabla$ with center $x$. Then $\left(\varphi_{*} \nabla\right)_{0} \in \bigwedge^{2} T_{0}^{*} \mathbf{R}^{m} \otimes$ $T_{0} \mathbf{R}^{m}$ modulo the obvious $G L\left(\mathbf{R}^{m}\right)$-invariant identification and $j_{0}^{1}\left(\varphi_{*} K\right) \in$ $J_{0}^{1}\left(T^{*} \mathbf{R}^{m} \otimes T \mathbf{R}^{m}\right)$. We put

$$
\left(\varphi_{*} \Delta_{c}(\nabla, K)\right)_{0}:=c\left(\left(\varphi_{*} \nabla\right)_{0}, j_{0}^{1}\left(\varphi_{*} K\right)\right) .
$$

If $\psi$ is another normal coordinate system of $\nabla$ with center $x$, then $\psi=\eta \circ \varphi$ for a $G L\left(\mathbf{R}^{m}\right)$-map $\eta$. Then $\left(\psi_{*} \Delta_{c}(\nabla, K)\right)_{0}=\left(\varphi_{*} \Delta_{c}(\Delta, K)\right)_{0}$ because of the $G L\left(\mathbf{R}^{m}\right)$-invariance of $c$. That is why, the definition of $\Delta_{c}(\nabla, K)$ is correct. Thus we have the $\mathcal{M} f_{m}$-natural operator $\Delta_{c}: Q_{\text {gen-af }} \rightsquigarrow\left(\otimes^{2} T^{*} \otimes\right.$ $T) \oplus\left(T^{*} \otimes T\right)$. Clearly, $\Phi\left(\Delta_{c}\right)=c$.

2. The main result. The main result of the note is the following "reduction" theorem.

Theorem 1. The problem of finding all $\mathcal{M} f_{m}$-natural operators $B: Q_{\text {gen-af }}$ $\rightsquigarrow Q_{\text {gen-af }}$ can be reduced to the one of describing all $G L\left(\mathbf{R}^{m}\right)$-invariant maps $\mathbf{R}^{m *} \otimes \mathbf{R}^{m} \rightarrow \otimes{ }^{k} \mathbf{R}^{m *} \otimes \otimes^{k} \mathbf{R}^{m}$ for $k=1,3$.

Proof. Any $G L\left(\mathbf{R}^{m}\right)$-invariant map $c:\left(\bigwedge^{2} \mathbf{R}^{m *} \otimes \mathbf{R}^{m}\right) \oplus\left(\mathbf{R}^{m *} \otimes \mathbf{R}^{m}\right) \oplus$ $\left(\otimes^{2} \mathbf{R}^{m *} \otimes \mathbf{R}^{m}\right) \rightarrow\left(\otimes^{2} \mathbf{R}^{m *} \otimes \mathbf{R}^{m}\right) \oplus\left(\mathbf{R}^{m *} \otimes \mathbf{R}^{m}\right)$ is the system of $G L\left(\mathbf{R}^{m}\right)$ invariant maps

$$
c_{1}:\left(\bigwedge^{2} \mathbf{R}^{m *} \otimes \mathbf{R}^{m}\right) \oplus\left(\mathbf{R}^{m *} \otimes \mathbf{R}^{m}\right) \oplus\left(\otimes^{2} \mathbf{R}^{m *} \otimes \mathbf{R}^{m}\right) \rightarrow \otimes^{2} \mathbf{R}^{m *} \otimes \mathbf{R}^{m}
$$


and

$$
c_{2}:\left(\bigwedge^{2} \mathbf{R}^{m *} \otimes \mathbf{R}^{m}\right) \oplus\left(\mathbf{R}^{m *} \otimes \mathbf{R}^{m}\right) \oplus\left(\otimes^{2} \mathbf{R}^{m *} \otimes \mathbf{R}^{m}\right) \rightarrow \mathbf{R}^{m *} \otimes \mathbf{R}^{m} .
$$

Using the invariance of $c_{i}$ with respect to the homotheties $a_{t}=t i d_{\mathbf{R}^{m}}$ for $t>0$, we obtain the respective homogeneity conditions. Then (by the homogeneous function theorems) $c_{1}\left(\Lambda, \tau_{0}, \tau_{1}\right)$ is linear in $\Lambda$ and $\tau_{1}$ and not necessarily linear in $\tau_{0}$. Then $c_{1}$ can be treated as the sum of $G L\left(\mathbf{R}^{m}\right)$-linear maps

$$
c_{1}^{\prime}: \mathbf{R}^{m *} \otimes \mathbf{R}^{m} \rightarrow\left(\bigwedge^{2} \mathbf{R}^{m *} \otimes \mathbf{R}^{m}\right)^{*} \otimes\left(\otimes^{2} \mathbf{R}^{m *} \otimes \mathbf{R}^{m}\right) \subset \otimes^{3} \mathbf{R}^{m *} \otimes \otimes^{3} \mathbf{R}^{m}
$$

and

$$
c_{1}^{\prime \prime}: \mathbf{R}^{m *} \otimes \mathbf{R}^{m} \rightarrow\left(\otimes^{2} \mathbf{R}^{m *} \otimes \mathbf{R}^{m}\right)^{*} \otimes\left(\otimes^{2} \mathbf{R}^{m *} \otimes \mathbf{R}^{m}\right) \tilde{=} \otimes^{3} \mathbf{R}^{m *} \otimes \otimes^{3} \mathbf{R}^{m} .
$$

By the same arguments, $c_{2}\left(\Lambda, \tau_{0}, \tau_{1}\right)$ is independent of $\Lambda$ and $\tau_{1}$. Then $c_{2}: \mathbf{R}^{m *} \otimes \mathbf{R}^{m} \rightarrow \mathbf{R}^{m *} \otimes \mathbf{R}^{m}$ is a $G L\left(\mathbf{R}^{m}\right)$-invariant map.

Now, Theorem 1 is an immediate consequence of Lemma 1.

\title{
REFERENCES
}

[1] Dębecki, J., The natural operators transforming affinors to tensor fields of type $(3,3)$, Acta Univ. Palacki. Olomuc., Fac. rer. nat., Mathematica 39 (2000), 37-49.

[2] Kobayashi, S., Nomizu, K., Foundations of Differential Geometry. Vol. I, J. WileyInterscience, New York-London, 1963.

[3] Kolář, I., Michor, P. W., Slovák, J., Natural Operations in Differential Geometry, Springer-Verlag, Berlin, 1993.

\author{
Jan Kurek \\ Włodzimierz M. Mikulski \\ Institute of Mathematics \\ Maria Curie-Skłodowska University \\ Institute of Mathematics \\ pl. M. Curie-Skłodowskiej 1 \\ Jagiellonian University \\ Lublin \\ S. Eojasiewicza 6 \\ Poland \\ Cracow \\ Poland \\ e-mail:kurek@hektor.umcs.lublin.pl e-mail:Wlodzimierz.Mikulski@im.uj.edu.pl
}

Received December 31, 2016 